\title{
Criteria for Instability and Chaos in Nonlinear Systems
}

\author{
Evgeny Nikolaevich Perevoznikov ${ }^{1}$, Henry Evgenievich Skvortsov ${ }^{2}$ \\ ${ }^{1}$ A.F. Mozhaisky Military Space Academy, St. Petersburg, Russia \\ ${ }^{2}$ St. Petersburg State University, St. Petersburg, Russia \\ Email: eperevoznikov@yandex.ru, Gskvortsov@yandex.ru
}

How to cite this paper: Perevoznikov, E.N. and Skvortsov, H.E. (2018) Criteria for Instability and Chaos in Nonlinear Systems. Journal of Applied Mathematics and Physics, 6, 382-388.

https://doi.org/10.4236/jamp.2018.62036

Received: June 13, 2017

Accepted: February 20, 2018

Published: February 23, 2018

Copyright $\odot 2018$ by authors and Scientific Research Publishing Inc. This work is licensed under the Creative Commons Attribution International License (CC BY 4.0).

http://creativecommons.org/licenses/by/4.0/

(c) (i) Open Access

\begin{abstract}
In the article, the methods of investigating the instability that were formulated earlier by the authors are systematized in the form of a set of criteria for the instability and chaos. The latest ones are used to study chaotic dynamics in the problems of Sprott and the nonlinear electronic generator of the CRC.
\end{abstract}

\section{Keywords}

Nonlinear Dynamics, Criteria of Instability and Chaos, The Problems of Chaotic Dynamics of Sprott and Nonlinear Electronic Generator

\section{Introduction}

In this article, the results of the theory of instability developed in [1]-[5], are supplemented and systematized in the form of a set of spectral methods and chaos criteria for three-dimensional dynamical systems. Limitation of three-dimensional systems allows reflecting the main aspects of the problem in a fairly simple form. The models which are used for the demonstration relate to essentially nonequilibrium dynamic systems with pronounced chaotic behavior.

\section{The Initial for the Analysis of Dynamics Is the Singular-Dynamic (SD-Method) and the Criteria of Instability and Chaos, (see [1] [3])}

The SD-method is based on the idea of a connection between mathematical features (Singularities) of dynamic equations with the change of dynamic regimes, with dynamic qualitative transitions and accompanying instabilities. The algorithm for using the method is:

a) First, the features of the expressions describing the model are determined. 
In our case these are the singularities of the right-hand sides of equations: zeros, infinities, bifurcation points, as well as the zeros of their derivatives, etc.;

b) Simplification of dynamic equations using singularities;

c) Analysis and solution of simplified equations, determination of the parameters of points of singularities;

d) The calculation of the criteria and the determination of the conditions for changing the regimes - the emergence of instability and chaos.

The system of equations of the described models has a general form

$$
d_{t} x=g_{1}(x, y, z) ; \quad d_{t} y=g_{2}(x, y, z) ; \quad d_{t} z=g_{3}(x, y, z)
$$

where $(x, y, z)$ are dynamic variables, $\left(x_{0}, y_{0}, z_{0}\right)$-the corresponding initial conditions. The right-hand sides of Equations (1) ( $g_{i}$-actions)-Nonlinear second-order functions by non-linearity.

The standard spectral method is in linearizing the system (1) relatively of stationary solutions-zeros of operations, obtaining the spectral equation and its analysis. In the process of analysis, complexes of the coefficients of the spectral equation are being obtained-Criteria determining the presence of instabilities and chaotic regimes.

Thus, writing down the linearized system in general form

$$
\partial_{t} \delta \boldsymbol{x}=\hat{E} \delta \boldsymbol{x} \rightarrow\left(\lambda \delta_{i k}-e_{i k}\right) \delta \boldsymbol{x}=0, e_{i k}=\frac{\mathrm{d} g_{i}}{\mathrm{~d} x_{k}} \equiv d_{k} g_{i} .
$$

Here $\hat{E}$-evolutionary matrix and its elements $e_{\alpha, \beta}$. The features of linear systems are contained in the spectrum, which is determined by the spectral equation (SE)

$$
S(\lambda)=\operatorname{det}\left(\lambda \delta_{i k}-e_{i k}\right)=0
$$

and which for the system of three equations-system (1) equally

$$
S(\lambda)=\lambda^{3}+s \lambda^{2}+p \lambda+q=0 .
$$

Coefficients $s, p, q$ depend on the parameters of the linearization point and are expressed through $e_{i k}$. In particular,

$$
s=e_{11}+e_{22}+e_{33}=-\operatorname{div} \boldsymbol{G} .
$$

The divergence values of the vector of the right-hand sides of Equations (2) indicates the areas of growth and reduction of the phase volume and is one of the conditions of stability-inconsistency. Depending on the sign $\operatorname{div} \boldsymbol{G}$ dynamics is called active ( $\operatorname{div} \boldsymbol{G}>0)$, neutral $(\operatorname{div} \boldsymbol{G}=0)$, dissipative ( $\operatorname{div} \boldsymbol{G}<0)$. In this case, the boundaries of these regions are also singular points in the spectrum.

Next we consider stationary points-zeros of right-hand sides. If, due to non-linearity, several stationary points are obtained, depending on the parameters of the system, one should also study their features.

The instability is due to the presence of the roots of the spectral equation-(SE) with a positive real part; chaos-with the presence of oscillations and the interaction of the growing and damped modes, i.e. The presence in the spectrum of 
a singular point of the saddle-focus. Then the spectral relations defining the boundaries of the regions of instability and chaos have the form [2]
a) $q \leq 0$ and $K_{1}=q-s p \geq 0$;
b) $p>0$;
c) $q \cdot K_{1}>0$

The first inequalities in (5) are the instability conditions, the second-reflects the presence of oscillations, the third-the saddle-shaped nature of the spectrum. Criteria (5) become obvious if we take into account the relationship between them and the coefficients of SE with eigenvalues, for SE (3) equal to

$$
\begin{aligned}
& q=-\lambda_{1} \cdot \lambda_{2} \cdot \lambda_{3}=-v_{1}\left(v_{2}^{2}+\omega^{2}\right), \\
& q-s p=2 v_{2}\left[\left(v_{1}+v_{2}\right)^{2}+\omega^{2}\right], \\
& p=\left(\lambda_{1} \cdot \lambda_{2}+\lambda_{1} \cdot \lambda_{3}+\lambda_{2} \cdot \lambda_{3}\right)=2 v_{1} \cdot v_{2}+\left(v_{2}^{2}+\omega^{2}\right), \\
& s=-\left(\lambda_{1}+\lambda_{2}+\lambda_{3}\right)=-\left(v_{1}+2 v_{2}\right) .
\end{aligned}
$$

where $\lambda_{1}=v_{1} ; \lambda_{2,3}=v_{2}+i \omega$ real and complex-conjugate roots SE (10).

Criteria (5) are thus the spectral criteria for instability and chaos.

The criterion that generalizes and complements the spectral ones is the L-criterion method. It consists in obtaining the neutrality conditions (the stability-instability boundary) through the coefficients of the dynamic equations using the determinant of the block matrix $\hat{L}$ composed of an evolution matrix $\hat{E}$ and its elements. The L-criterion formula has the general form [2] [4]

$$
(-1)^{n+1} \cdot \operatorname{det}(\widehat{L})=(-1)^{n+1} \cdot \operatorname{det}\left[\delta_{\alpha, \beta} \widehat{E}+\widehat{I}_{\alpha, \beta}^{*}\right]=\prod_{\alpha, \beta=1}^{n}\left(\tilde{\lambda}_{\alpha}+\tilde{\lambda}_{\beta}^{*}\right) \frac{>^{H y} 0}{<_{y} 0} \text {. }
$$

where $\hat{E}$-evolutionary matrix, $e_{\alpha, \beta}^{*}$-complex conjugate elements of the ma$\operatorname{trix} \hat{E}, \hat{I}$ - unit matrix, $n$-dimension of the system, $\tilde{\lambda}_{i}=\partial_{t}\left(\ln \left|\delta a_{i}\right|\right)$-Generalized eigenvalues of the evolution matrix (which are an analog of Lyapunov exponents and coincide with them in the case of stationary states).

The equality of the criterion (7) to zero corresponds to the presence of zero derivatives (eigenvalues), a change in the sign of the criterion-the change in the sign of the time derivatives in dynamic equations. Thus, the analysis of the factors in (7) is an analysis of the eigenvalue spectrum of the evolution matrix of the nonlinear system and, consequently, the analysis of the signs of time derivatives in the linearized dynamic Equations (2).

As an example of the application of criteria, we consider two problems that explicitly demonstrate unstable chaotic regimes:

a) one of the models of chaotic dynamics - the Sprott model [6];

b) Model of a nonlinear electronic generator Kiyashko S.V., Pikovsky A.S., Rabinovich M.I. (CRC) [6].

\section{Equations of the Sprott Model Have the Form}

$$
\partial_{t} x=x y-z \equiv g_{1} ; \quad \partial_{t} y=x-y \equiv g_{2} ; \quad \partial_{t} z=x+a z \equiv g_{3} .
$$

where $x, y, z$-dynamic variables, $a$-parameter.

Indicator of type of dynamics $\operatorname{div} G=(y-1+a)$. Depending on the choice of 
the initial condition $-y_{0}$ it is possible to get all three kinds of chaos

System (8) has two stationary points

$$
s t 1 \Rightarrow(x, y, z)_{s}=(0,0,0) ; \quad s t 2 \Rightarrow(x, y, z)_{s}=\left(-\frac{1}{a},-\frac{1}{a}, \frac{1}{a^{2}}\right) .
$$

For the Sprott model, linearized near arbitrary states $(x, y, z)$ system (2), corresponding evolutionary matrix $E$ and also the spectral equation with coefficients have the form

$$
\begin{gathered}
\partial_{t} \delta \boldsymbol{x}=\hat{E} \delta \boldsymbol{x}, \quad \hat{E}=\left(\begin{array}{ccc}
y & x & -1 \\
1 & -1 & 0 \\
1 & 0 & a
\end{array}\right) \\
S(\lambda)=\lambda^{3}+s \lambda^{2}+p \lambda+q=0 \\
s=1-a-q, \quad p=-x+(1-y) \cdot(1-a), \quad q=1+a(x+y)
\end{gathered}
$$

For the first stationary point, the roots of SE are equal

$$
\lambda_{1}=-1, \quad \lambda_{2,3}=\frac{a}{2} \pm \sqrt{\frac{a^{2}}{4}-1}
$$

Spectral criteria-(5), L-criterion-(7) and dynamics index-(4) accordingly give expressions

$$
\begin{gathered}
q=1>0, \quad q(q-s p)=1-(1-a)^{2} \geq 0, \quad \operatorname{det} L=8 a^{2}(a-1) \cdot(a-2) \\
\operatorname{div} \boldsymbol{G}=(a-1) \geq 0 .
\end{gathered}
$$

For the second stationary point (because of the awkwardness of analytical expressions), we give the numerical values of roots and criteria

$$
\begin{gathered}
a \in(0.1-0.3) \cup(a>4.5), \lambda_{1}>0, \lambda_{2,3}<0 ; \\
a \in(0.3-4.5), \lambda_{1}>0, \lambda_{2,3}=r \pm i \omega, r<0 . \\
q=-1<0, q(q-s p)<0, \operatorname{det} L>0, \operatorname{div} \boldsymbol{B}>0(\text { npu } a>1.62)
\end{gathered}
$$

From the conditions (12 - 15) it follows that both stationary states are unstable for any values of the parameter $a$, chaotic oscillations exist in limited intervals.

For the first stationary point with $a \in(0-2)$, the second $-a \in(0.3-4.5)$. With $a>2$ Both steady states are nondissipative i.e. the phase volume of the system increases.

The criteria and the spectrum are consistent with each other.

\section{The Nonlinear Electronic Generator CRC}

Such generators with a substantially nonlinear (N-shaped) volt-ampere characteristic of a tunnel diode refer to nonequilibrium dynamic systems in which excitation of unstable chaotic oscillations is observed [6].

The CRC generator was proposed and studied in the experimental regime by Kiyashko S.V., Pikovsky A.S., Rabinovich M.I. [6]. The electrical circuit of a generator with a nonlinear volt-ampere characteristic and an attractor reflecting the dynamics of chaotic oscillations are shown in Figure 1, Figure 2. 


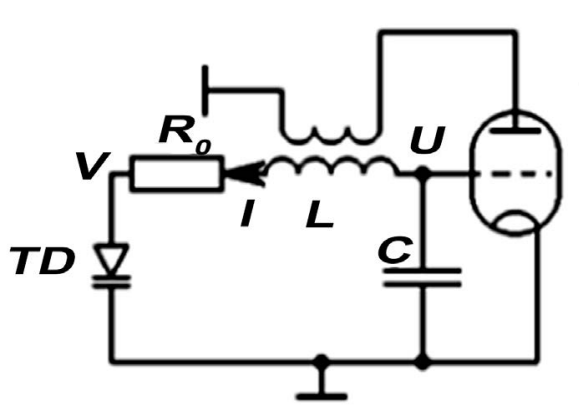

(a)

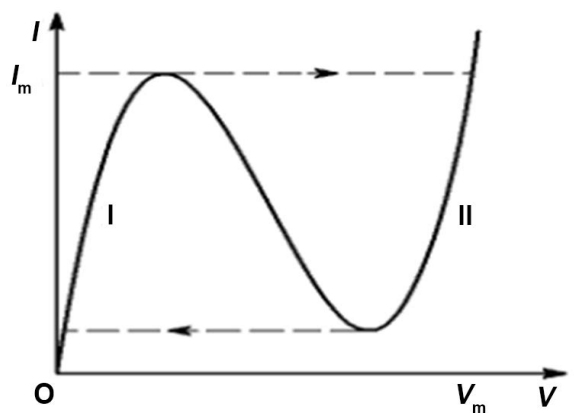

(b)

Figure 1. Scheme and volt-ampere characteristic of an electronic generator with a nonlinear tunnel diode.

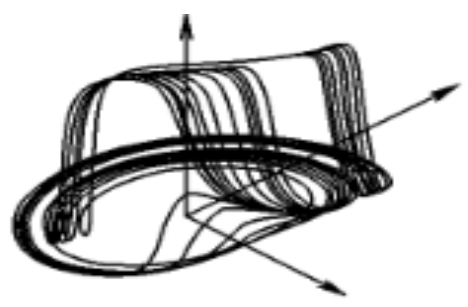

Figure 2. The phase portrait of an attractor in the space of dynamical variables $(\mathrm{x}, \mathrm{y}, \mathrm{z})$.

The initial dynamical equations of the generator have the form [6]

$$
L d_{t} I=U-V+I R ; \quad C d_{t} U=-I ; \quad c d_{t} V=I_{m} f\left(V / V_{m}\right)=I ，
$$

where $I$-current in the circuit, $U$-voltage , $V$-diode voltage, $L$-inductance, $C$-chain capacity, $c$ - tunnel diode capacitance, $(-R)-$ Negative diode resistance (gain), $I_{m}, U_{m}$-maximum values. After the introduction of dimensionless variables

$$
x=I / I_{m} ; \quad y=U I_{m} / \sqrt{C / L} ; \quad z=V / V_{m} ; \quad \tau=t / \sqrt{L C},
$$

the initial system of dynamical equations in dimensionless form takes the form

$$
\begin{aligned}
& d_{\tau} x=a x+y-b z=g_{x} \\
& d_{\tau} y=-x=g_{y} \\
& d_{\tau} z=e(x-g(z))=g_{z}
\end{aligned}
$$

where

$$
a=R \sqrt{C / L} ; \quad b=\left(V_{m} / I_{m}\right) \sqrt{C / L} ; \quad e=C / b c \gg 1 ; g=z\left(14.4 z^{2}-22 z+8.6\right) .
$$

Thus, using the SD method and the criteria (5), (7), we obtain the conditions for instability and chaos of the dynamic regimes of the generator described by the system (18) in the analytical form in terms of the dynamic coefficients (19).

a) First, the features of the expressions describing the model are determined. In our case, these are the singularities of the right-hand sides of the equationszeros (stationary points) and the zeros of their derivatives (extremes of the voltampere characteristic). 
The system (18) has one stationary point corresponding to the absence of current

$$
x_{s}=y_{s}=z_{s}=0,
$$

and two extremum points

$$
d_{z} g=43.2\left(z^{2}-1.02 z+0.2\right)=0 ; \quad z_{1}=0.265, \quad z_{2}=0.755 .
$$

Values $g(z)$ at these points, respectively, equal to 1 and 0.151 , one of these points corresponds to a maximum another to a minimum of the function $\mathrm{g}$.

The values of the divergence of the vector of the right-hand sides of equations (3) indicating the regions of growth and decrease of the phase volume are

$$
\operatorname{div} \boldsymbol{G}=a-e d_{z} g
$$

and depends on the position of the point on the volt-ampere characteristic.

For a nonlinear generator, the system (18) linearized near arbitrary states and the corresponding evolution matrix $E$ have the form

$$
\partial_{t} \delta \boldsymbol{x}=\hat{E} \delta \boldsymbol{x}, \quad \hat{E}=\left(\begin{array}{ccc}
2 h & 1 & -b \\
-1 & 0 & 0 \\
e & 0 & -e d_{z} g
\end{array}\right)
$$

And the spectral coefficients are, respectively,

$$
s=e d_{z} g-2 h, \quad p=\left(1+e b-2 h e d_{z} g\right), \quad q=d_{z} g .
$$

The conditions (5) after simple transformations in the general case take the form

$$
\begin{aligned}
& 2 h\left[\left(2 h-e d_{z} g\right)\left(e b / 2 h-e d_{z} g\right)+1\right] \geq 0, \\
& 3\left(1+e b-2 h e d_{z} g\right)=\left(e d_{z} g-2 h\right)^{2}, \\
& 2 h e d_{z} g\left[\left(2 h-e d_{z} g\right)\left(e b / 2 h-e d_{z} g\right)+1\right]>0
\end{aligned} .
$$

The first of these expressions is the condition of neutrality (the stability boundary), the second is the condition for the presence of oscillations (the existence of a frequency), and the third is the presence of a saddle-focus point in the spectrum.

From the conditions (25) it follows that at the initial point (20) the system is dissipative, unstable, does not have chaotic oscillations.

At the extremum points $\left(d_{z} g=0\right)$ The conditions (25) are respectively equal to

$$
\begin{aligned}
& 2 h\left[\left(2 h-e d_{z} g\right)\left(e b / 2 h-e d_{z} g\right)+1\right]=0 \rightarrow 2 h(e b+1)>0, \\
& 3\left(1+e b-2 h e d_{z} g\right)=\left(e d_{z} g-2 h\right)^{2} \rightarrow 3(e b+1)=4 h^{2}, \\
& 2 h e d_{z} g\left[\left(2 h-e d_{z} g\right)\left(e b / 2 h-e d_{z} g\right)+1\right]>0 \rightarrow 0
\end{aligned} .
$$

Whence follows the presence of unstable chaotic oscillations near these points, which corresponds to unstable chaotic regimes observed in experiments with nonlinear generators [1].

Application of L-criterion (8) to the system (18) gives the expression 


$$
\begin{aligned}
\operatorname{det} L= & 2^{5} h^{2}\left(-e d_{z} g\right)\left[\left(2 h-e d_{z} g\right)\left(e b / 2 h-e d_{z} g\right)+1\right] \\
& \cdot\left[\left(2 h-e d_{z} g\right)\left(4 h-e d_{z} g\right)-(e b+1)\right]
\end{aligned}
$$

It follows from the comparison of the L-criterion (27) with conditions (25) that it contains both the neutrality condition and the saddle-focus condition and indicates the possibility of a third critical regime-the third factor in (27). The signs of the three factors in (27) correspond to the signs of the time derivatives in the dynamical system (18), and the combination of signs indicates the possibility in the generator of an unstable chaotic regime.

In conclusion, we note that the main result of the work consists in the formulation and demonstration of simple effective methods and criteria for chaos. The examples considered are the model problem of Sprott and a nonlinear electronic generator of Kiyashko S.V. Pikovsky A.S., Rabinovich M.I., which show the clarity and simplicity of the criteria and allows constructively identify chaotic behavior in three-dimensional problems with any kind of actions, and also generalize to systems of higher dimension.

The instability and chaos conditions obtained in studies of dynamic regimes in nonlinear generators can be useful both for further theoretical studies of dynamic regimes in nonlinear generators and for optimizing the operation of devices using such generators.

Note that with regard to the very phenomenon of chaos, there are enough problems that are yet to be solved. For example, questions about the time of development of chaos, the alternation of modes-intermittency, the amplitude of chaotic oscillations, the frequency spectrum, etc. But these are questions of the following studies.

\section{References}

[1] Perevoznikov, E.N. and Skvortsov, H.E. (2014) Fizicheskaya neustoychivost' i kachestvennyye perekhody. Materialy X miedzynarodwej naukowi-praktycznej konferencji. Польша, Przemysl май, 30, 79-84.

[2] Skvortsov, H.E. and Perevoznikov, E.N. (2014) Dinamicheskiye kachestvennyye perekhody, Materialy X miedzynarodwej naukowi-praktycznej konferencji. Evropeyskaya nauka XX1 veka (Pol sha, Przemys), 31, 82-84.

[3] Perevoznikov, E.N. (2006) Metody analiza ustoychivosti neravnovesnykh system. Izv. Vuzov, Fizika, 10, 34-39.

[4] Skvortsov, H.E. and Perevoznikov, E.N. (2015) Singulyarno-dinamicheskiye kriterii neustoychivosti i khaosa, Mezhdunarodnyy nauchno-issledovatel'skiy zhurnal. Fiziko-Matematicheskiye Nauki, 9, 91-93.

[5] Perevoznikov, E. and Mikhailova, O. (2015) Neutrality Criteria for Stability Analysis of Dynamical Systems through Lorentz and Rossler Model Problems. Journal of Applied Mathematics and Physics, 5, 569-576.

[6] Kuznetsov, S.P. (2006) Dinamicheskiy Khaos. 356 c. 